\title{
Accuracy of a Fluoroscopy Technique for Assessing Patellar Tracking
}

T.S.Y. Tang ${ }^{1}$, N.J. MacIntyre ${ }^{2}$, H.S. Gill ${ }^{3}$, R.A. Fellows ${ }^{2}$, N.A. Hill ${ }^{2}$, D.R. Wilson ${ }^{4}$, and R.E. Ellis ${ }^{1,2}$

1 School of Computing, Queen's University, Kingston, Ontario, Canada

\{ttang,ellis\}@cs.queensu.ca

2 Department of Mechanical Engineering, Human Mobility Research Center, Queen's University, Kingston, Ontario, Canada

3 Nuffield Department of Orthopaedic Surgery/OOEC, Nuffield Orthopaedic Center,

Oxford University, Oxford, UK

4 Department of Orthopaedics, University of British Columbia,

Vancouver, B. C., Canada

\begin{abstract}
Accuracy of a novel fluoroscopy-based method of assessing patellar tracking was determined by comparing the pattern of patellar motion with respect to orientation (flexion, internal rotation, and lateral tilt) and translation (lateral, proximal, and anterior) with the pattern of patellar motion measured using Roentgen Stereophotogrammetric Analysis (RSA) in one cadaver knee specimen. Each pose in the patellar motion could be obtained from a single as well as multiple calibrated fluoroscopic images. The mean error (SD) varies from $0.73(0.44)$ to $1.60(0.48)$ degrees for patellar orientation and from $0.48(0.37)$ to 1.20 (0.57) $\mathrm{mm}$ for patellar translation. These errors appear to be sufficiently low to identify clinically significant differences in patellar kinematics.
\end{abstract}

\section{Introduction}

Patellofemoral Pain Syndrome (PFPS) is a common knee disorder among physically active adolescents and young adults [3]. It is characterized by an atraumatic onset of anterior knee pain. This pain is aggravated by activities such as running, squatting, climbing stairs, or sitting with the knee flexed for long periods of time.

Controversy exists regarding the cause of PFPS and, therefore, the optimal treatment for this condition is not clear. A prevailing theory is that PFPS results when the patella is moving abnormally or maltracking in the trochlear groove of the femur during knee flexion and extension. It stands to reason that when the bones in the lower limb are not lined up in an ideal way, abnormal gliding at the patellofemoral joint (PFJ) may occur. Cadaver studies have shown that malalignment, simulated by changing the normal quadriceps angle, can lead to shifts in the location of the load in the PFJ [6] and clinical evidence of lower limb malalignment is observed in individuals with PFPS [3]. However, findings of patellar and lower limb malalignment are also observed in individuals with no 
complaints of knee pain [7. Moreover, clinical tests and imaging techniques used to measure 2D alignment of the patella and lower limb in static positions are unable to assess patellar tracking, a 3D movement. Measures of patellar tracking have been obtained in vivo using Roentgen Stereophotogrammetric Analysis (RSA) and a variety of motion analysis systems; however, the methods used are either highly invasive 9815 or involve surface markers that are prone to movement artifacts or inconsistent placement [10. Because of the limitations and variability of measurement techniques used, the causative role of patellar maltracking in PFPS has not been established.

Recently magnetic resonance imaging (MRI) has been used to assess patellar tracking but the dimension of the magnet bore limits the range and type of knee flexion activity that can be performed [11. Fluoroscopic imaging has been used to measure patellar motion during weightbearing activity [12]; however, the fluoroscopic images were not corrected for deformation or loss of 3D information. The ultimate goal for such methods is to measure 3D patellar kinematics during activities that typically cause symptoms in individuals with PFPS in order to establish normative values and to determine if patterns of patellar tracking in individuals with PFPS differ from the norm. To this end, we are developing an assessment tool for measuring 3D patellar kinematics during loaded knee flexion based on corrected fluoroscopic images. To assess the accuracy of our method, we compared the fluoroscopy-based measures of patellar kinematics to those obtained using the established RSA method.

\section{Methods}

\subsection{Specimen Preparation}

Tantalum beads with a diameter of $0.8 \mathrm{~mm}$ (Tilly Medical Products AB, Sweden) were implanted in a fresh frozen human cadaver right knee (19 year-old male, ScienceCare Anatomical, Phoenix, AZ) with intact soft tissue. Six to eight beads were inserted into each of the femur, patella, and tibia, such that the beads are clearly separated and widely distributed to avoid symmetry. A high-resolution CT scan with a slice thickness of $1.25 \mathrm{~mm}$ was performed to determine the surface model of each of the bones, and coordinate systems were assigned to each bone [5]. The 3D locations of the tantalum beads were determined in CT coordinates.

\subsection{Measurement of Patellar Tracking Using Fluoroscopy}

Figure 1 depicts the knee secured in a loading rig that held the femur in a fixed horizontal position and controlled the degree of knee flexion by altering the length of a cord sutured to the quadriceps tendon. Thus a load was applied to the patellofemoral joint as the knee was flexed through a range of approximately 0 to 75 degrees.

A series of fluoroscopic images (anterior-posterior, left anterior oblique, and right anterior oblique views) were obtained from eight static positions of knee 


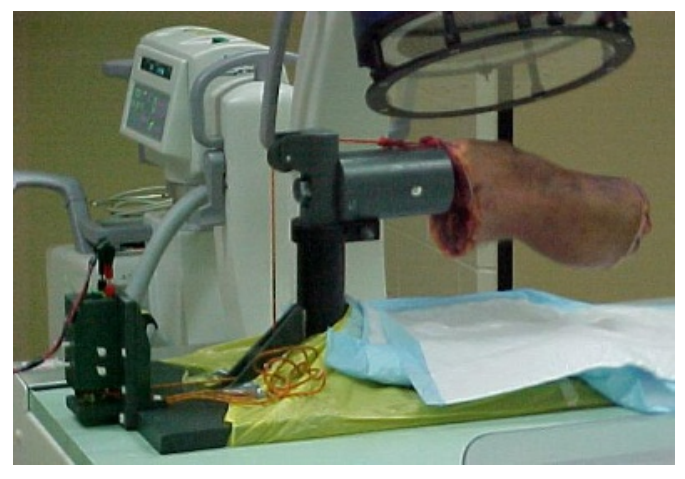

(a)

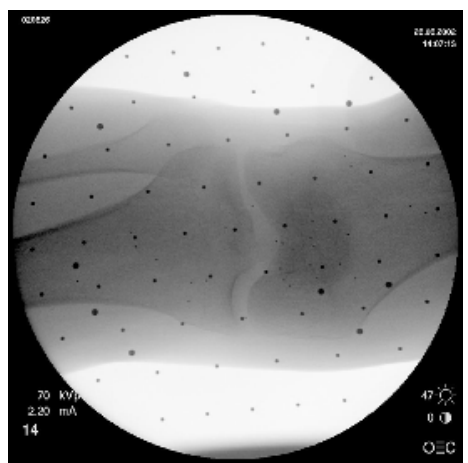

(b)

Fig. 1. Fluoroscopic imaging of a cadaver knee mounted in the loading rig with the calibration drum secured to the image intensifier (a) and an uncalibrated fluoroscopic image showing marker and reference beads (b).

flexion using a fluoroscope (OEC 9800, General Electric, USA). The fluoroscopic images were calibrated as they were taken to remove spatial distortion in the images and to locate the position of the x-ray source [14. This was done by placing a calibration drum containing two parallel layers of reference markers of known size and position (iGo Technologies, Canada) on the image intensifier of the fluoroscope.

\subsection{Image Registration}

For each of the bones, the $2 \mathrm{D}$ fiducial locations were determined from the calibrated fluoroscopic images, and were registered with the $3 \mathrm{D}$ fiducial locations from the CT scan such that the $3 \mathrm{D}$ pose of the bones were reconstructed. 2D$3 \mathrm{D}$ registration was done using a variant of the iterative closest point (ICP) algorithm 213. The algorithm uses the 2D locations of the fiducial markers to construct a set of back-projected lines to the $3 \mathrm{D}$ x-ray source location. Occasionally one or two fiducial markers were occluded by the reference markers. Since a minimum of four fiducial markers are required for the algorithm, this occlusion rate could be tolerated. The pose of the bone is then calculated by minimizing the distance between the $3 \mathrm{D}$ locations of the markers in the bone and their corresponding back-projected lines. Although one could use a single fluoroscopic image to determine the pose of the knee, all fluoroscopic images of a particular knee pose could be used to increase the accuracy of the registration. In this case, the registration results from each single image were used as an initial guess, then a second step was done by fixing the relative poses of the bones among the images when minimizing the overall back-projection error.

Using the registration results, the position of the patella relative to the femur was then described using a joint coordinate system (JCS) to determine variables 
describing patellar orientation (flexion, internal rotation, and lateral tilt) and translation (lateral, proximal, and anterior) through approximately $75^{\circ}$ of knee flexion [5].

\subsection{Ground Truth}

To establish the ground truth for patellar tracking measures, we performed the same procedure using RSA [1. A biplanar technique was used to acquire a pair of orthogonal x-ray images for each pose. The knee was mounted in the test rig described above and placed in front of a planar calibration cage containing control and fiducial markers (Tilly Medical Products AB, Sweden). The two xray tubes were oriented at 30 degrees from the perpendicular and positioned 60 inches from the x-ray films secured behind the calibration cage. Pairs of x-ray images were acquired at ten positions of flexion as the loaded knee was flexed from full extension to about 75 degrees.

\section{Results}

Data obtained using all fluoroscopic images of each knee pose, and RSA data describing patellar orientation and translation were plotted as a function of knee flexion angle and splines were fit to both sets of data (Figure 2). For a given position of knee flexion, the values were compared to determine the error, and the mean absolute error and standard deviation was calculated for each orientation and translation variable (Table 1).

The accuracy of the fluoroscopy-based method was also determined using a single image of each knee pose. For comparison, these results are summarized in Table 2,

\section{Discussion}

The fluoroscopy-based method successfully measured 3D patellar tracking using planar fluoroscopic images. The accuracy of the fluoroscopy method of measur-

Table 1. Mean absolute errors and standard deviations for variables describing patellar orientation and translation through loaded knee flexion using multiple fluoroscopic images of each knee pose.

\begin{tabular}{|l|c|c|}
\hline Patella Motion & Mean Error & Standard Deviation \\
\hline Orientation (degrees) & 1.49 & 0.39 \\
Flexion & 1.60 & 0.48 \\
Internal Rotation & 0.73 & 0.44 \\
Lateral Tilt & & \\
\hline Translation (mm) & 0.48 & 0.37 \\
Lateral & 0.53 & 0.38 \\
Proximal & 1.20 & 0.57 \\
Anterior & \\
\hline
\end{tabular}



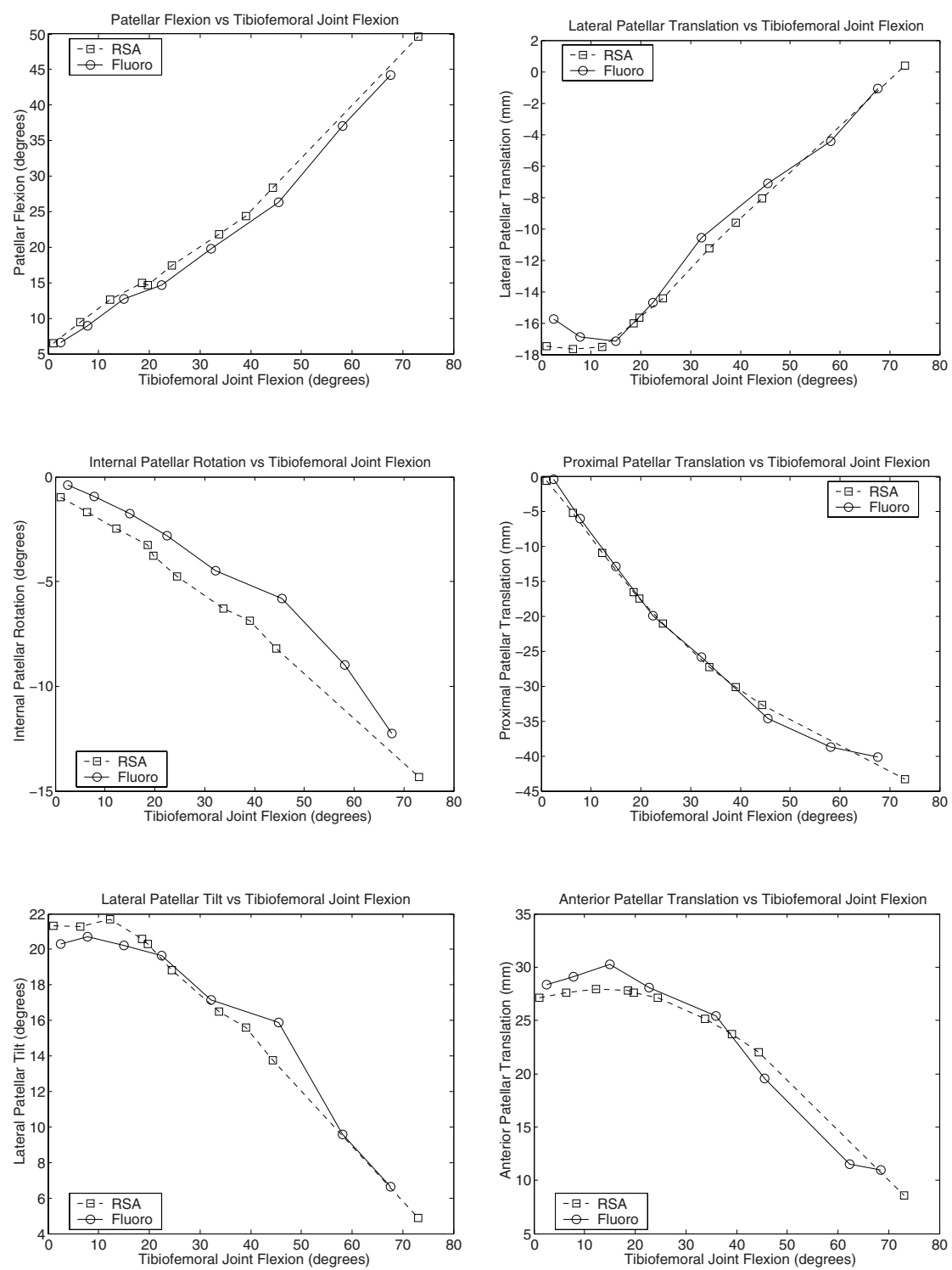

Fig. 2. Results of the patella orientation (left column) and translation (right column) using fluoroscopy (solid) and RSA (dashed).

ing patellar tracking in loaded knee flexion was poorer comparing to the RSA method, which has a reported error of about 0.6 degrees and $0.25 \mathrm{~mm} \mathrm{[1}$. We believe the major source of error in our method lies in the accuracy of fluoroscopic image calibration. The location of the x-ray source and the corrected location of each fiducial marker directly affect the back-projection of the markers by which 
the orientation and position of each bone is determined. However, the measurement errors are comparable to the reported accuracy of an MRI-based method for measuring PFJ kinematics 4], and appear to be sufficiently low, particularly for patellar orientation, to identify clinically significant differences in patterns of patellar tracking. With a better calibration technique, it is possible to use a single fluoroscopic image of each knee pose to determine the PFJ kinematics.

The main disadvantage of both methods is that they are invasive as fiducial markers have to be implanted. We are currently studying the use of markerless registration techniques in measuring PFJ kinematics with this fluoroscopy technique. Preliminary results using intensity-based registration techniques showed that the accuracy was similar to the fiducial-based method but intensity-based techniques are not as versatile because the patella is clearly visible only in mediallateral views.

The fluoroscopy-based method of measuring PFJ kinematics has two key advantages over the RSA method. First, it does not require a static calibration cage and two x-ray tubes, instead it uses a portable imaging system (C-arm), which greatly improve the applicability. Second, because fluoroscopy can record dynamic activity, the fluoroscopic technique can be applied to measure patellar tracking during continuous knee movement. Once the intensity-based technique is established, this method will permit the assessment of 3D patellar kinematics during activities that typically cause symptoms in individuals with PFPS.

\section{Conclusion}

Planar fluoroscope images of the PFJ obtained through a range of loaded knee flexion can be used to accurately assess patellar tracking. The method described in this paper may be used to investigate the effect of malalignment or surgical intervention strategies on patellar tracking in cadaver specimens. Future applications will include the assessment of patterns of patellar tracking in order to discriminate individuals with PFPS from those without and to investigate the effectiveness of conservative and surgical interventions aimed at correcting patellar alignment.

Table 2. Mean absolute errors and standard deviations for variables describing patellar orientation and translation through loaded knee flexion using a single fluoroscopic image of each knee pose.

\begin{tabular}{|l|c|c|}
\hline Patella Motion & Mean Error & Standard Deviation \\
\hline Orientation (degrees) & & \\
Flexion & 1.71 & 0.64 \\
Internal Rotation & 1.75 & 0.64 \\
Lateral Tilt & 0.84 & 0.55 \\
\hline Translation (mm) & & \\
Lateral & 0.99 & 0.81 \\
Proximal & 0.51 & 0.35 \\
Anterior & 2.09 & 1.35 \\
\hline
\end{tabular}


Acknowledgements. This research was supported by the Canadian Institutes of Health Research and the Natural Sciences and Engineering Research Council of Canada. NJM is supported by The Arthritis Society/CIHR Health Research Partnership Fund Postdoctoral Fellowship. The CT, fluoroscopic and x-ray images were acquired with the assistance of Marg Bollen, Charge Technologist, Department of Imaging Services at Kingston General Hospital, Canada.

\section{References}

1. J. Alfaro-Adrian, H. S. Gill, and D. W. Murray. A comparison of charnley elite and exeter femoral stems using rsa. Journal of Bone and Joint Surgery - British Volume, 81(1): 130-134, 1999.

2. Paul J. Besl and Neil D. McKay. A method for registration of 3-D shapes. IEEE Transactions on Pattern Analysis and Machine Intelligence, 14(2): 239-256, February 1992.

3. J. C. Fairbank, P. B. Pynsent, J. A. van Poortvliet, and H. Phillips. Mechanical factors in the incidence of knee pain in adolescents and young adults. Journal of Bone and Joint Surgery - British Volume, 66: 685-693, 1984.

4. R. A. Fellows, N. A. Hill, N. J. MacIntyre, S. C. Leclaire, T. S. Y. Tang, M. M. Harrison, R. E. Ellis, and D. R. Wilson. Accuracy of a magnetic resonance imagingbased method for assessing three-dimensional patellar tracking in vivo. In Transactions of the 49th Annual Meeting, volume 28. Orthopaedic Research Society, 2003.

5. E. S. Grood and W. J. Suntay. A joint coordinate system for the clinical description of t hree-dimensional motions: applications to the knee. Journal of Biomechanics, 105: 136-144, 1994.

6. H. H. Huberti and W. C. Hayes. Patellofemoral contact pressures. Journal of Bone and Joint Surgery - American Volume, 66, 1984.

7. L. L. Johnson, G. E. van Dyke, J. R. Green, A. W. Pittsley, B. Bays, S. M. Gully, and J. M. Phillips. Clinical assessment of asymptomatic knees: comparison of men and women. Journal of Arthroscopy, 14: 347-359, 1998.

8. T. J. Koh, M. D. Brabiner, and R. J. de Swart. In vivo tracking of the human patella. Journal of Bicomechanics, 25(6): 637-643, 1992.

9. M. A. LaFortune, P. R. Cavanagh, H. J. Sommer III, and A. Kalenak. Threedimensional kinematics of the human knee during walking. Journal of Biomechanics, 25(4): 347-357, 1992.

10. D. K. Ramsey and P. F. Wretenberg. Biomechanics of the knee: methodological considerations in the in vivo kinematic analysis of the tibiofemoral and patellofemoral joint. Clinical Biomechanics, 14(9): 595-611, 1999.

11. F. T. Sheehan and J. E. Drace. Quantitative $\mathrm{mr}$ measures of three-dimensional patellar kinematics as a research and diagnostic tool. Medicine and Science in Sports Exercise, 31: 1399-1405, 1999.

12. L. A. Stein, A. N. Endicott, J. S. Sampalis, M. A. Kaplow, M. D. Patel, and N. S.Mitchell. Motion of the patella during walking: A video digital-fluoroscopic study in healthy volunteers. American Journal of Radiology, 161: 617-620, 1993.

13. T. S. Y. Tang, R. E. Ellis, and G. Fichtinger. Fiducial registration from a single X-Ray image: a new technique for fluoroscopic guidance and radiotherapy. In Medical Image Computing and Computer-Assisted Intervention - MICCAI 2000, pages 502-511. Springer, 2000. 
14. Thomas S. Y. Tang. Calibration and point-based registration of fluoroscopic images. Master's thesis, Queen's University, Kingston, Ontario, Canada, 1999.

15. S. A. Varess, F. G. Lippert, M. C. Y. Hou, and T. Takamoto. Patellar tracking patterns measurement by analytical x-ray photogrammetry. Journal of Biomechanics, 12: 639-650, 1979. 